\title{
Scalar correlators near the 3-flavor thermal critical point
}

\section{Xiao-Yong Jin ${ }^{* * a}$, Yoshinobu Kuramashi ${ }^{a b c}$, Yoshifumi Nakamura ${ }^{a d}$, Shinji Takeda ${ }^{a e}$, and Akira Ukawa ${ }^{a}$}

${ }^{a}$ RIKEN Advanced Institute for Computational Science, Kobe, Hyogo 650-0047, Japan

${ }^{b}$ Graduate School of Pure and Applied Sciences, University of Tsukuba, Tsukuba, Ibaraki 305-8571, Japan

${ }^{c}$ Center for Computational Sciences, University of Tsukuba, Tsukuba, Ibaraki 305-8577, Japan

${ }^{d}$ Graduate School of System Informatics, Department of Computational Sciences, Kobe University, Kobe, Hyogo 657-8501, Japan

${ }^{e}$ Institute of Physics, Kanazawa University, Kanazawa 920-1192, Japan

${ }^{\dagger}$ Email: xjin@anl.gov

Present address: Argonne Leadership Computing Facility, Argonne National Laboratory, Argonne, Illinois 60439, USA

We investigate screening masses at both sides of the first order finite temperature transition with 3 quark flavors using the nonperturbatively improved clover fermion action and the Iwasaki gauge action. We have developed the method of hierarchical truncations with stochastic probing to accelerate the noise estimator for evaluating quark loops at every spatial lattice slices. At parameter values we study, the flavor singlet scalar meson has a screening mass about half of the pion screening mass. It becomes lighter as the system approaches the critical endpoint.

The 32nd International Symposium on Lattice Field Theory

23-28 June, 2014

Columbia University New York, NY

* Speaker. 


\section{Introduction}

QCD with three or more massless quark flavors would have a first order finite temperature transition [1]. With increasing quark masses, the first order transition ends at a second order critical point, where the correlation length of the flavor singlet scalar meson diverges [2].

Lattice QCD provided evidences [3, 4] of small singlet scalar meson screening masses, by simulations of the naive staggered fermion action, using a temporal extend, $N_{t}=4$. Since then, no new simulations have been exploring this area of research, partly because of the increasing difficulties of locating first order thermal transitions with improved staggered fermion formulations.

Simulating 3-flavor finite temperature lattices using the nonperturbatively improved clover fermion action and the Iwasaki gauge action, we are able to locate the first order transition with lattice sizes of $24^{3} \times 8$ and $32^{3} \times 8$. This gives us the opportunity of studying the screening masses near the critical endpoint on the first order transition line.

\section{Measurement techniques}

We measure the connected screening propagators on three spatial directions, using multiple point sources either with or without Gaussian smearing, and point sinks. We use Gaussian smearing parameters, $r=4$ and $n=100$, so the source vector centered at $x_{0}$ is

$$
\phi(x)=\left(1+\frac{r^{2}}{4 n} \nabla^{2}\right)^{n} \delta\left(x-x_{0}\right),
$$

where

$$
\nabla^{2} \chi(x)=\sum_{i=1,2,3} U_{i}(x) \chi(x+\hat{i})+U_{i}^{\dagger}(x-\hat{i}) \chi(x-\hat{i})-2 \chi(x) .
$$

The flavor singlet screening correlator after zero momentum projection for $N_{f}$ quark flavors,

$$
\begin{aligned}
\left\langle\sum_{q}[\bar{q}(x) q(x)] \sum_{q}[\bar{q}(y) q(y)]\right\rangle & =N_{f}^{2}\langle\bar{\psi} \psi\rangle^{2}+A\left(e^{-m|x-y|}+e^{-m\left|x-y-N_{l}\right|}\right)+\cdots \\
& =-N_{f}\left\langle\operatorname{tr}\left[S(x, y) S^{\dagger}(y, x)\right]\right\rangle+N_{f}^{2}\langle\operatorname{tr}[S(x, x)] \operatorname{tr}[S(y, y)]\rangle,
\end{aligned}
$$

has a nonzero vacuum state due to finite chiral condensate, and requires evaluating disconnected diagrams. We call the exponential decay part the screening propagator, and write it as

$$
\begin{aligned}
e^{-m|x|}+e^{-m\left|N_{l}-x\right|} & \propto-C(x)+N_{f} D(x), \\
C(x) & =\left\langle\operatorname{tr}\left[S\left(x_{0}, x_{0}+x\right) S^{\dagger}\left(x_{0}+x, x_{0}\right)\right]\right\rangle \\
D(x) & =\left\langle\operatorname{tr}\left[S\left(x_{0}, x_{0}\right)\right] \operatorname{tr}\left[S\left(x_{0}+x, x_{0}+x\right)\right]\right\rangle-\left\langle\operatorname{tr}\left[S\left(x_{0}, x_{0}\right)\right]\right\rangle^{2} \\
& =\left\langle\left(\operatorname{tr}\left[S\left(x_{0}, x_{0}\right)\right]-\langle\bar{\psi} \psi\rangle\right)^{2}\right\rangle-\left\langle\frac{1}{2}\left(\operatorname{tr}\left[S\left(x_{0}, x_{0}\right)\right]-\operatorname{tr}\left[S\left(x_{0}+x, x_{0}+x\right)\right]\right)^{2}\right\rangle
\end{aligned}
$$

where averaging over $x_{0}$ is implicit for brevity, $N_{l}$ is the spatial extent of the lattice, $S$ is the unsmeared quark propagator with $\left\langle\operatorname{tr}\left[S\left(x_{0}, x_{0}\right)\right]\right\rangle=\langle\bar{\psi} \psi\rangle$, and $C$ and $D$ are the connected and disconnected parts of the propagator respectively. The separation of the two terms in Eq. 2.8 makes them sensitive to the statistical noise from evaluating $\operatorname{tr}[S(x, x)]$. They help us determine the required statistics and the computational cost for our method detailed below. 


\begin{tabular}{c|rrrrrrrr}
$d_{\min }$ & 1 & 2 & 3 & 4 & 5 & 6 & 7 & 8 \\
No. of diluted vectors & 1 & 2 & 23 & 16 & 120 & 210 & 411 & 256 \\
$\sigma_{\text {imp }} / \sigma$ & 1.14 & 0.82 & & 0.58 & & & & 0.28
\end{tabular}

Table 1: Number of space-time diluted vectors for a $32^{3} \times 8$ lattice corresponding to different values of $d_{\min }$, and the resulting improvement ratio of standard deviations. $\sigma_{\text {imp }}$ is the standard deviation of $\langle\bar{\psi} \psi\rangle$ computed with spin-color separation and space-time dilution; $\sigma$ is from spin-color combined undiluted stochastic sources. Standard deviations are estimated at the same computational cost at a fixed CG iteration number of 25 .

We use complex $Z(2)_{\Re} \times Z(2)_{\mathfrak{I}}$ volume random sources, applying the idea of probing-instead of diluted uniform sources in the reference [5], we use stochastic sources with space-time dilution using the greedy multi-coloring algorithm. We test a few configurations with different values of $d_{\min }$, which is the minimum number of links between nonzero sites in a diluted vector. We also combine it with spin-color separation [6], for the extra benefit verified in our tests. Table 1 shows a representative test result, for a particular gauge configuration of a lattice size $32^{3} \times 8$. While increasing $d_{\min }$, the number of space-time diluted vectors increases exponentially with the exception of $d_{\min }=2,4$, and 8 , which give relatively small number of diluted vectors due to symmetry. For $d_{\min }=1$, which means no space-time dilution, spin-color separation alone does not help in reducing the standard deviation for the same cost. Using spin-color separation and space-time dilution together, we find increasingly smaller standard deviations with increasing $d_{\min }$ at the same computational cost.

The improvement factor of four (with $d_{\min }=8$ in table 1 ) comes if we solve $256 \times 12$ vectors for one configuration, which is impractical. The truncated solver method (TSM) [7] brings us further improvements when we extend it to use multiple levels of conjugate gradient (CG) truncations and apply spin-color separation and space-time dilution with different $d_{\min }$ at different truncation levels hierarchically. This is our method of hierarchical truncations with stochastic probing (HTwSP).

One possible caveat: at a fixed CG iteration before converging, given the same initial guess, space-time dilutions with different $d_{\min }$ produce different solutions. Combining results from different $d_{\min }$ at different CG truncation levels would potentially contain bias.

To test how big the difference is, we compare the undiluted results from 49152 volume random sources, and the diluted results from 256 source vectors per spin-color with space-time dilution of $d_{\min }=4$, which is also 49152 vectors $(=256 \times 12 \times 16)$. Figure 1 shows $\langle\bar{\psi} \psi\rangle$ at each spatial slices for one tested configuration of an ordered-start ensemble with a lattice size of $32^{3} \times 8, \kappa=0.14085$, and $\beta=1.73$. The figure shows results from the first eight $C G$ iterations with a zero vector as the initial guess. Results from these two schemes are different at finite CG iterations. This difference, however, appears to decay exponentially. At a few hundred iterations where truncated results are used in this study, though untested, the difference should be well below statistical errors or even machine precision, so that no bias can be introduced in this way.

For the HTwSP scheme in this study, we pick a handful of gauge configurations and search for the best combinations of CG truncation levels and values of $d_{\min }$ for each level, while respecting our cost constraints and statistical precision needed for our study. Table 2 shows the best parameters we find on one of the test configurations, for the original one level TSM, two level and three level 


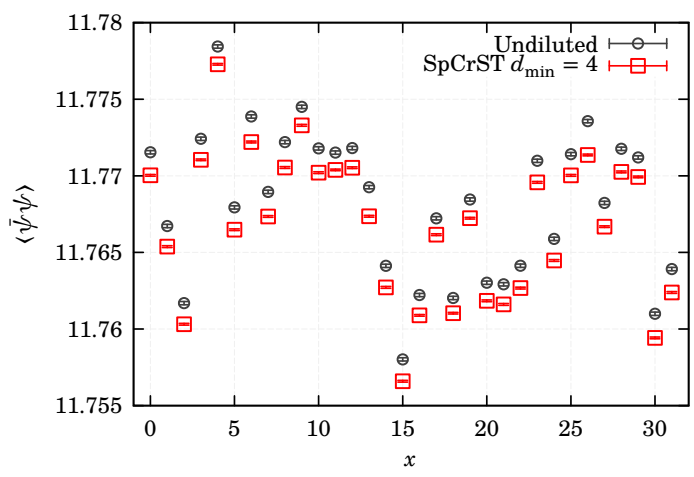

(a) Results at the eighth CG iteration

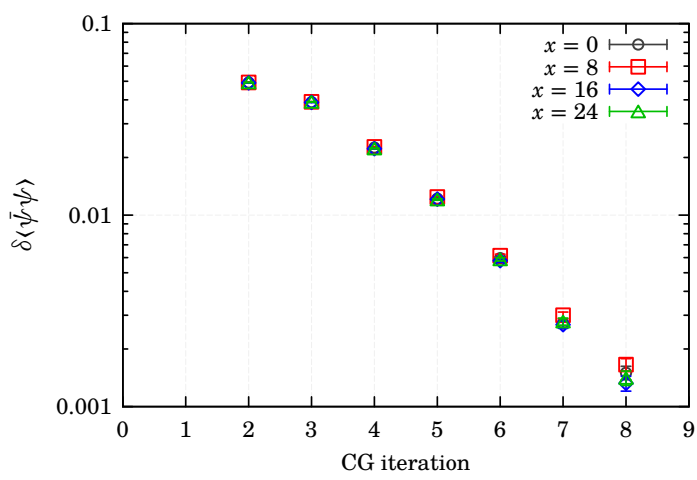

(b) Difference as a function of CG iteration

Figure 1: Results from the first eight CG iterations using undiluted random sources and diluted random sources of spin-color separation and space-time dilution with $d_{\min }=4$.

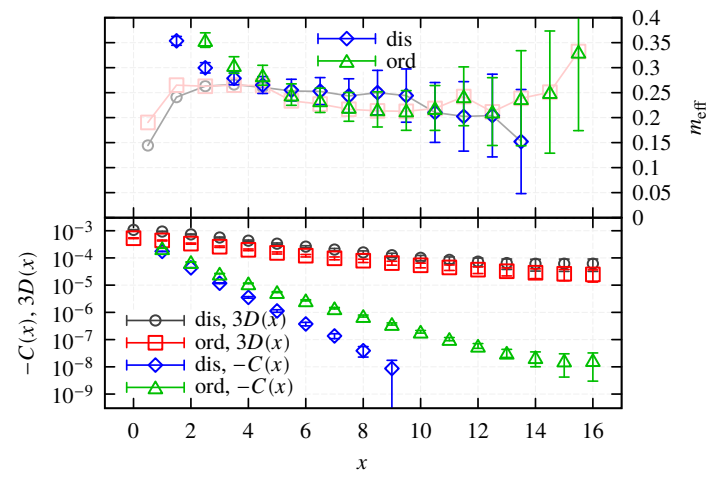

(a) $\beta=1.73, \kappa=0.14085$

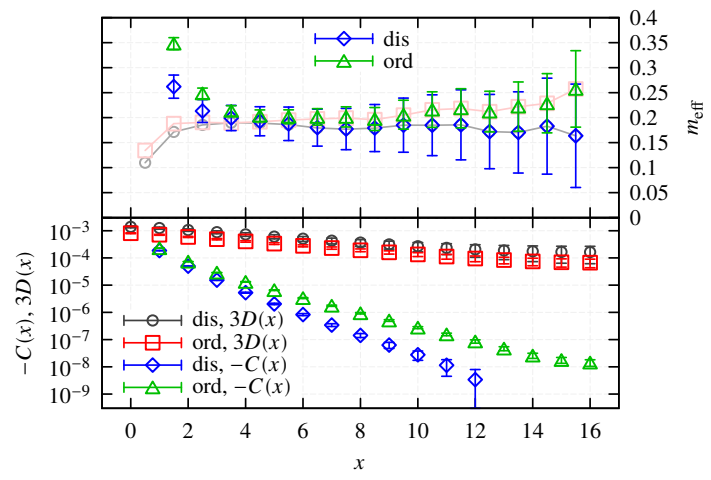

(b) $\beta=1.74, \kappa=0.14054$

Figure 2: Lower panels show the connected and disconnected screening propagators of the flavor singlet scalar meson. Upper panels show the effective mass obtained from the full propagators, and the ones from disconnected propagators without error bars using light color as comparison. In the keys, 'dis' stands for the disordered-start ensemble, and 'ord' stands for the ordered-start ensemble.

TSM (2L-TSM, 3L-TSM), and two level HTwSP. We try to measure and minimize the computed ratios of variances, $R_{\mathrm{imp}}=\sigma_{\mathrm{imp}}^{2} / \sigma^{2}$, improved against the undiluted one. For a fixed cost equivalent to about 500 convergent $\mathrm{CG}$ inversions, we find more than 40 times speedup using the scenario of 2L-HTwSP. Further speedup is guaranteed with a larger cost constraint.

\section{Simulation results}

We start simulations with ordered and disordered gauge configurations with lattice sizes of $24^{3} \times 8$ and $32^{3} \times 8$. For our two lattice coupling values of $\beta=1.73$ and 1.74 , we have tested a few $\kappa$ values and located the two-states signal at $\kappa=0.14085$ and 0.14054 respectively. Table 3 shows simple ensemble statistics. We measure screening propagators every 20 trajectories.

Figure 2 shows the connected and disconnected parts of the flavor singlet scalar meson propagator and its effective masses as a solution to propagator values at nearby two spatial separations. 


\begin{tabular}{lrrrr} 
Scenario & TSM & 2L-TSM & 3L-TSM & 2L-HTwSP \\
Cost & 500.3 & 493.2 & 486.2 & 501.9 \\
\hline$R_{\text {imp }}$ & $0.203(4)$ & $0.119(4)$ & $0.102(3)$ & $0.0231(6)$ \\
\hline$N_{\mathrm{h}}$ & 99 & 21 & 5 & 1 \\
Iter & 2049 & 2049 & 2049 & 2049 \\
$C_{\mathrm{h}}$ & 1 & 1 & 1 & 24 \\
\hline$N_{\mathrm{l}}$ & 2990 & 1312 & 442 & 4 \\
Iter $_{1}$ & 275 & 425 & 475 & 475 \\
$C_{1}$ & 1 & 1 & 1 & 192 \\
\hline$N_{\mathrm{l} 2}$ & & 8200 & 2964 & 1 \\
Iter $_{12}$ & & 50 & 150 & 200 \\
$C_{\mathrm{l} 2}$ & & 1 & 1 & 3072 \\
\hline$N_{\mathrm{l} 3}$ & & & 13255 & \\
Iter $_{13}$ & & & 25 & \\
$C_{13}$ & & & 1 &
\end{tabular}

Table 2: Improvements of different number of truncation levels, and one particular HTwSP scheme. Parameters are constrained by the computational cost. A 'Cost' of 1 equals computational cost of one convergent CG inversion. $R$ is the ratio of variances (improved against undiluted one), which equals the inverse of the speedup in the wall-clock time for a fixed statistical error. While the subscripts denote different truncation levels, parameters for each level are: $N$ is the number of random vector sets, the number of undiluted stochastic sources; $C$ is the number of random vectors in one set, counting in dilution cost; 'Iter' is the number of fixed CG iterations on that truncation level (2049 is the convergent iteration number).

\begin{tabular}{rrllrlll}
$\beta$ & $\kappa$ & $N_{l}$ & start & $N_{\text {conf }}$ & plaquette & gauge & polyakov \\
\hline 1.73 & 0.14085 & 24 & ord & 2930 & $0.528741(67)$ & $1.24940(19)$ & $0.00831(16)$ \\
1.73 & 0.14085 & 32 & ord & 13875 & $0.528701(34)$ & $1.249514(98)$ & $0.008315(56)$ \\
\hline 1.73 & 0.14085 & 24 & dis & 4060 & $0.51495(15)$ & $1.28832(43)$ & $0.002555(73)$ \\
1.73 & 0.14085 & 32 & dis & 16815 & $0.514765(55)$ & $1.28883(16)$ & $0.002480(22)$ \\
\hline 1.74 & 0.14054 & 32 & ord & 16000 & $0.529832(44)$ & $1.24603(13)$ & $0.009558(32)$ \\
\hline 1.74 & 0.14054 & 32 & dis & 10450 & $0.520610(98)$ & $1.27195(28)$ & $0.003606(65)$
\end{tabular}

Table 3: The expectation values of plaquette, gauge density, and Polyakov loop. Number of configurations has first 1000 removed as thermalization.

Because the disconnected part are orders of magnitude larger than the connected part, at long spatial separations, the effective masses from the full singlet propagator and the effective masses from the disconnected part alone agree. We see no statistical difference between the screening masses from disordered-start and ordered-start ensembles, which respectively correspond to the zero temperature and the finite temperature phases.

Figure 3 shows meson screening masses at the transition. The flavor singlet scalar $\sigma$ meson is about half as light as the pion, and continues to decrease as the system approaches the critical 


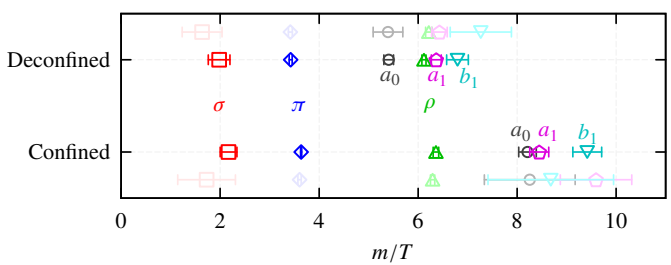

(a) $\beta=1.73, \kappa=0.14085$

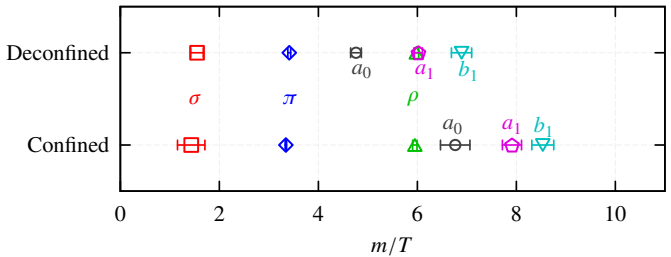

(b) $\beta=1.74, \kappa=0.14054$

Figure 3: Screening mass hierarchy across the first order transition. Symbols with light color represent results from $24^{3} \times 8$ lattices, while the rest are from $32^{3} \times 8$ lattices.

endpoint, from $\beta=1.73$ to $\beta=1.74$. In the two parameter sets we have, the screening masses of the $\rho$, the $\pi$, and the $\sigma$ mesons are almost constant across the transition. We see the pion screening mass is smaller in the deconfined phase than the confined phase at $\beta=1.73, \kappa=0.14085$, while it is in an opposite order in the other parameter set. This is different from previous results [3, 4] with staggered fermions. As the system undergoes the first order transition from confined zero temperature phase to deconfined finite temperature phase, the $a_{1}$ meson becomes degenerate with the $\rho$ meson, and the $a_{0}$ meson drops and becomes closer to the $\pi$ meson, as expected.

Near the second order critical point, the system should exhibit the behavior of critical scalings. The flavor singlet scalar meson screening masses should scale as the inverse of the correlation length, $m_{\sigma} \propto t^{v}$, as proposed by Gavin et al. [2]. Other observables, we expect, should scale as a combination of the magnetic like order parameter, $\propto t^{\bar{\beta}}$ (we write it as $\bar{\beta}$ to avoid confusion with the lattice coupling), and the energy like quantities, $\propto t^{-\alpha}$. Since we lack enough data sets to differentiate different universality classes, we treat the data as the mean field theory, such that $\alpha=0$ and $\bar{\beta}=v=1 / 2$. With the choice of the reduced temperature $t=\left(\beta-\beta_{\mathrm{C}}\right) / \beta_{\mathrm{C}}$, where $\beta_{\mathrm{C}}$ is the lattice coupling at the critical point, if the system is sufficiently close to the critical point, $m_{\sigma}^{2}$ should be linear in $\beta$, and the squared discontinuities of the plaquette, the gauge density, and the chiral condensate $\langle\bar{\psi} \psi\rangle$, should also be linear in $\beta$. Figure 4 shows these squared quantities, with linear lines going through two data points of each observable. Given the noisy scalar singlet meson screening masses, our extrapolations give consistent $\beta_{\mathrm{C}}$. The mean field theory thus predicts that the critical endpoint is between $\beta=1.745$ and 1.75 , which is consistent with our results from analysis of kurtosis intersections [8]. However, we need more data sets to determine the correct universality class.

\section{Summary}

We locate the first order transition with two-states signal at two parameter sets using the nonperturbatively improved clover fermion action and the Iwasaki gauge action.

We developed an easy-to-implement method of hierarchical truncations with stochastic probing, which gives us an estimated $40 \times$ speedup in measuring the quark condensate at spatial lattice slices.

Our data show the $\sigma$ screening mass is about half of the pion mass on both sides of the first order transition close to the endpoint. A preliminary extrapolation to the critical endpoint shows consistency between our data and the mean field critical scaling. To determine the correct universality class requires more statistics and expanded parameter sets. 


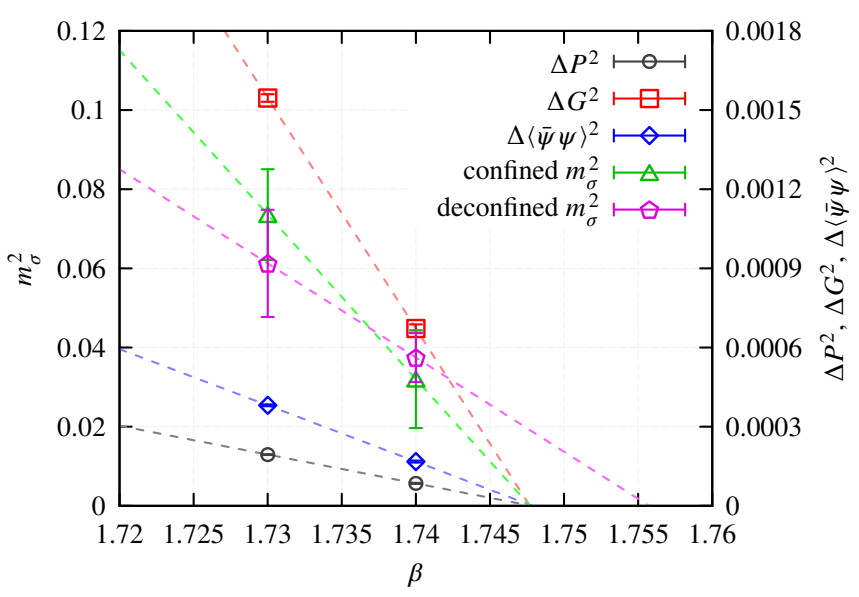

Figure 4: Linear extrapolations of $m_{\sigma}^{2}$ and the squared discontinuities of plaquette, gauge density, and $\langle\bar{\psi} \psi\rangle$.

\section{Acknowledgments}

The numerical calculations have been done on $\mathrm{K}$ computer at RIKEN AICS. Our lattice evolutions uses BQCD, while measurements use my modified code shared by the lattice group at the University of Helsinki.

This work is supported in part by the Grants-in-Aid for Scientific Research from the Ministry of Education, Culture, Sports, Science and Technology (No. 26800130).

\section{References}

[1] R. D. Pisarski and F. Wilczek, Remarks on the Chiral Phase Transition in Chromodynamics, Phys.Rev. D29 (1984) 338-341.

[2] S. Gavin, A. Gocksch, and R. D. Pisarski, QCD and the chiral critical point, Phys.Rev. D49 (1994) 3079-3082, [hep-ph/9311350].

[3] JLQCD Collaboration Collaboration, S. Aoki et al., Phase structure of lattice QCD at finite temperature for (2+1) flavors of Kogut-Susskind quarks, Nucl.Phys.Proc.Suppl. 73 (1999) 459-461, [hep-lat/9809102].

[4] X. Liao, Study of 3 flavor QCD finite temperature phase transition with staggered fermions, Nucl.Phys.Proc.Suppl. 106 (2002) 426-428, [hep-lat / 0111013$].$

[5] J. M. Tang and Y. Saad, A probing method for computing the diagonal of a matrix inverse, Numer. Linear Algebra Appl. 19 (May, 2012) 485-501.

[6] W. Wilcox, Noise methods for flavor singlet quantities, hep-lat/9911013.

[7] S. Collins, G. Bali, and A. Schafer, Disconnected contributions to hadronic structure: a new method for stochastic noise reduction, PoS LAT2007 (2007) 141, [arXiv: 0709.3217 ].

[8] X.-Y. Jin, Y. Kuramashi, Y. Nakamura, S. Takeda, and A. Ukawa, Critical endpoint of finite temperature phase transition for three flavor QCD, arXiv: 1411.7461. 